\title{
Xylene Recognition in Flexible Porous Coordination Polymer by Guest-dependent Structural Transition
}

\author{
Ping Wang, ${ }^{\dagger}$ Takashi Kajiwara, ${ }^{\dagger}$ Ken-ichi Otake, ${ }^{*}, \dagger$ Ming-Shui Yao, ${ }^{\dagger}$ Hirotaka Ashitani, ${ }^{\ddagger}$ Yoshiki \\ Kubota, ${ }^{\ddagger}$ and Susumu Kitagawa ${ }^{*} \dagger$ \\ †nstitute for Integrated Cell-Material Sciences (WPI-iCeMS), Kyoto University Institute for Advanced \\ Study, Kyoto University, Yoshida Ushinomiya-cho, Sakyo-ku, Kyoto 606-8501, Japan \\ $\$$ Department of Physical Science, Graduate School of Science, Osaka Prefecture University, 1-1 \\ Gakuen-cho, Naka-ku, Sakai, 1-1 Gakuen-cho, Naka-ku, Sakai, Osaka 599-8531, Japan
}

*E-mail: ootake.kenichi.8a@kyoto-u.ac.jp

*E-mail: kitagawa@icems.kyoto-u.ac.jp

\section{Experimental Procedures}

\subsection{Materials and Methods}

All chemicals were used as received without any further purification. 2,5-Dihydroxyterephthalic acid and 2-nitrobenzyl bromide, $o$-xylene, $m$-xylene and $p$-xylene were purchased from Tokyo Chemical Industries (TCl). Deuterated solvents for nuclear magnetic resonance (NMR) spectroscopy were purchased from Cambridge Isotope Laboratories. All solvents and other materials were purchased from either Nacalai Tesque or Wako Pure Chemicals.

${ }^{1} \mathrm{H}-\mathrm{NMR}$ spectra were measured at $25^{\circ} \mathrm{C}$ on Brucker models Ultrashild 500 Plus NMR spectrometer, where chemical shifts ( $\delta$ in ppm) were determined with a residual proton of the solvent as standard. Thermogravimetric analyses (TGA) were performed on a Rigaku model Thermo plus EVO with a heating rate of $5{ }^{\circ} \mathrm{C} / \mathrm{min}$ under $\mathrm{N}_{2}$ flow. Powder X-ray diffraction (PXRD) data were recorded on a Rigaku SmartLab X-ray diffractometer using Cu-K a radiation $(\lambda=1.54178 \AA)$ by depositing powder on a glass substrate, from $2 \theta=5^{\circ}$ up to $30^{\circ}$ with $0.02^{\circ}$ increment. The synchrotron PXRD data were collected using a synchrotron X-ray and multiple MYTHEN detectors of the BL02B2 beamline at Super Photon ring (SPring-8) by capillary transmission $(\lambda=0.79967 \AA)$.

Gas sorption experiments were carried out at $195 \mathrm{~K}$ using BELSORP mini gas adsorption instrument (MicrotracBEL, Japan). Vapor sorption isotherm measurements were conducted by using BELSORP-MAX instrument (MictrotracBEL, Japan).

Single crystals were picked with paraton oil on the tip of glass fiber and mounted on a Rigaku XtaLAB P200 diffractometer equipped with Dectoris PILATUS $200 \mathrm{~K}$ detector and Mo-Ka radiation $(\lambda=0.71073 \AA)$ whose temperature was controlled by liquid nitrogen. Crystal structures were solved by direct methods and refined by full-matrix least-squares cycles in $S H E L X$ 2017/1[1] using Rigaku CrystalStructure ver. 4.3 or Yadokari-XG software package. All non-hydrogen atoms were refined using anisotropic thermal parameters. The refinement results are summarized in Table S1. Crystallographic data for the crystal structures in CIF format has been deposited in the Cambridge Crystallographic Data Centre (CCDC) under deposition numbers CCDC-2081398-2081400. The data can be obtained free of charge via www.ccdc.cam.ac.uk/data_request/cif (or from the Cambridge Crystallographic Data Centre, 12 Union Road, Cambridge CB2 1EZ, U.K.) 


\section{Supporting Figures}

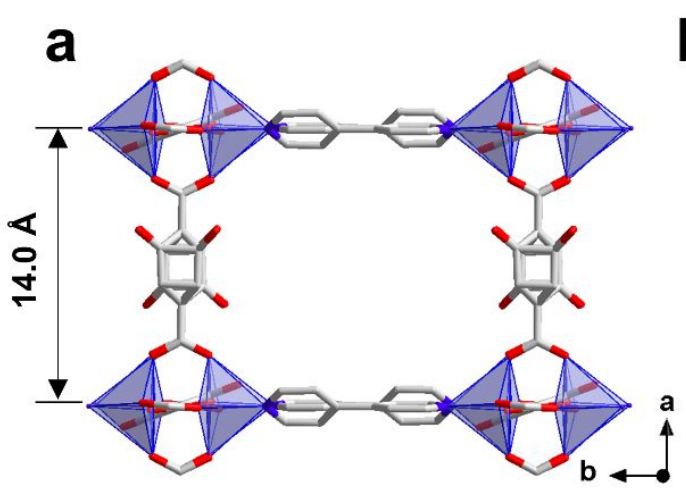

b
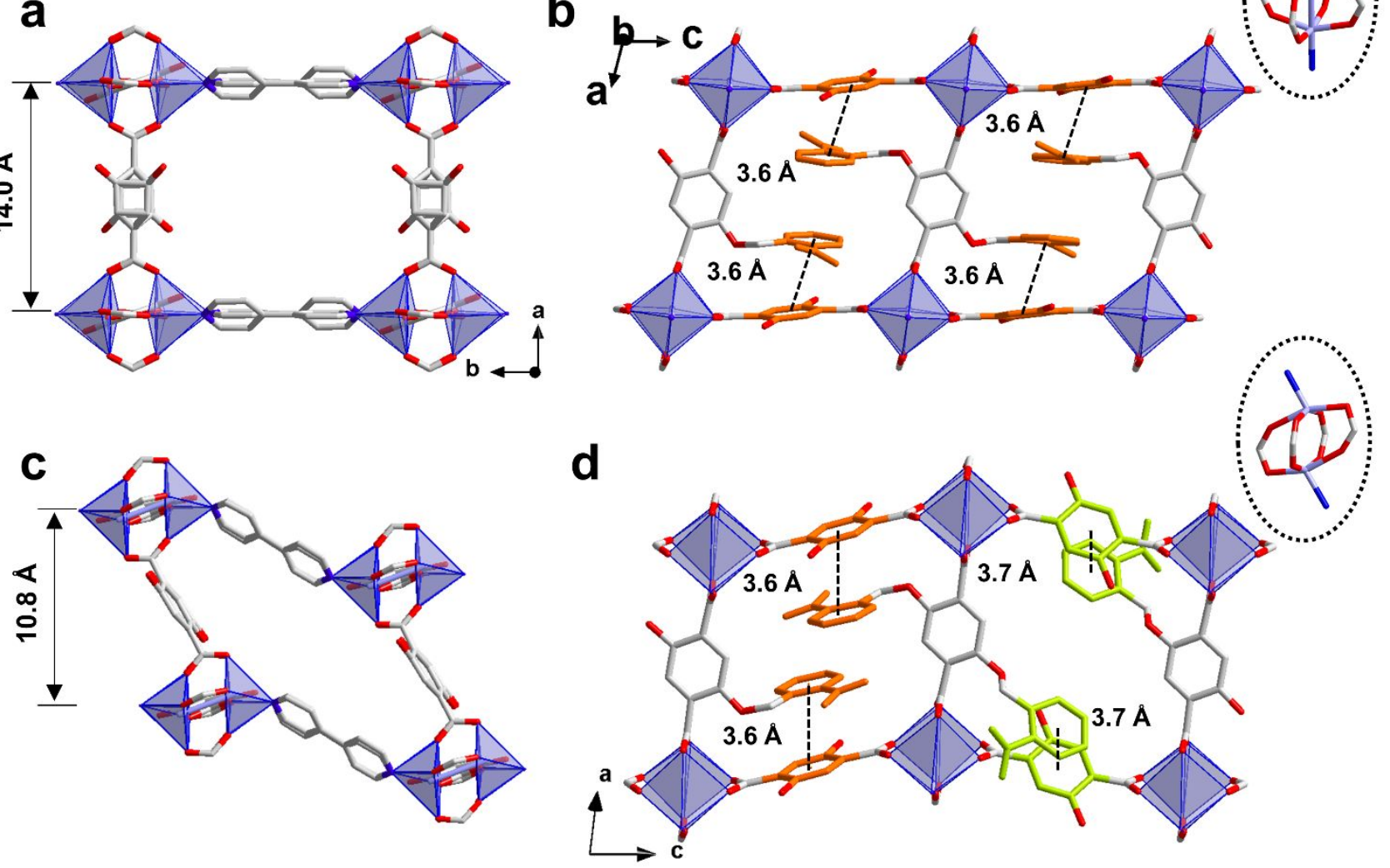

Figure S1. Structure of (a, b) PCP-1-as and (c, d) PCP-1-act. 


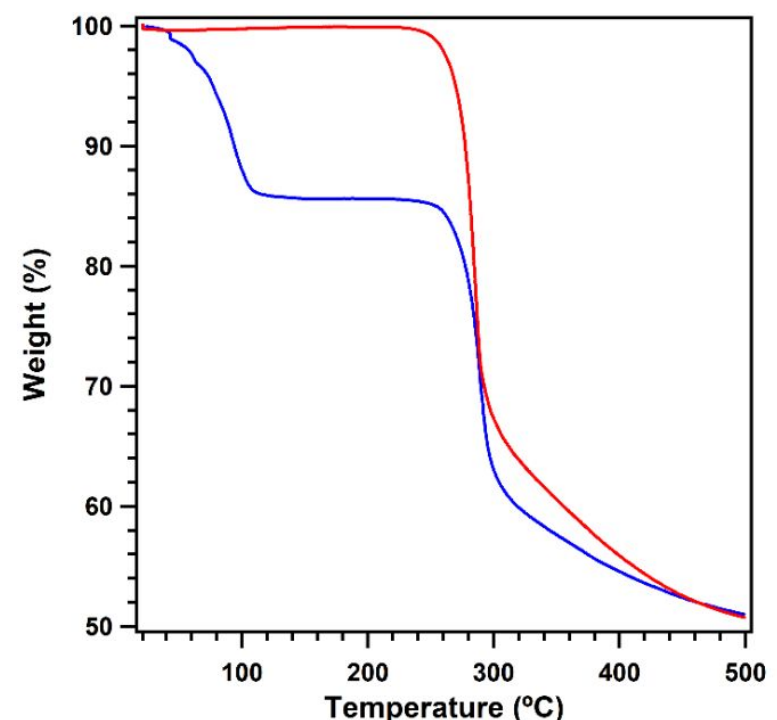

Figure S2. TGA curve of PCP-1-as (blue) and thermal activated PCP-1-act (red) 


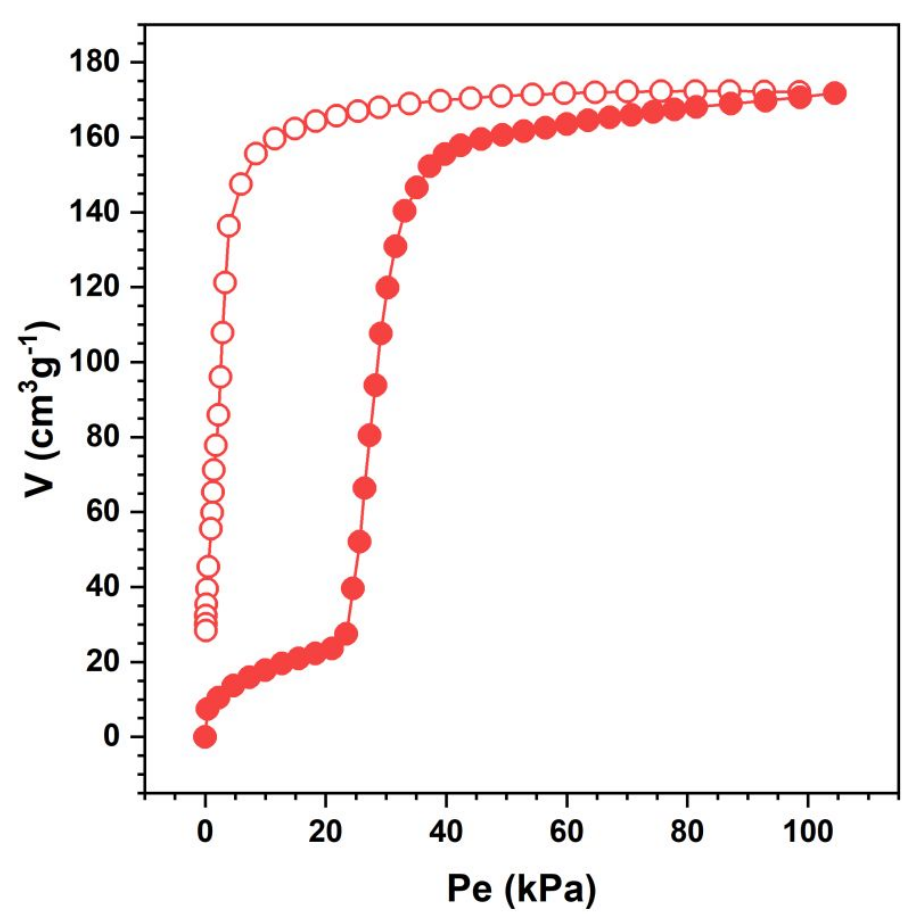

Figure S3. $195 \mathrm{~K} \mathrm{CO}_{2}$ sorption isotherm of PCP-1-act. Filled for adsorption and blank for desorption. 


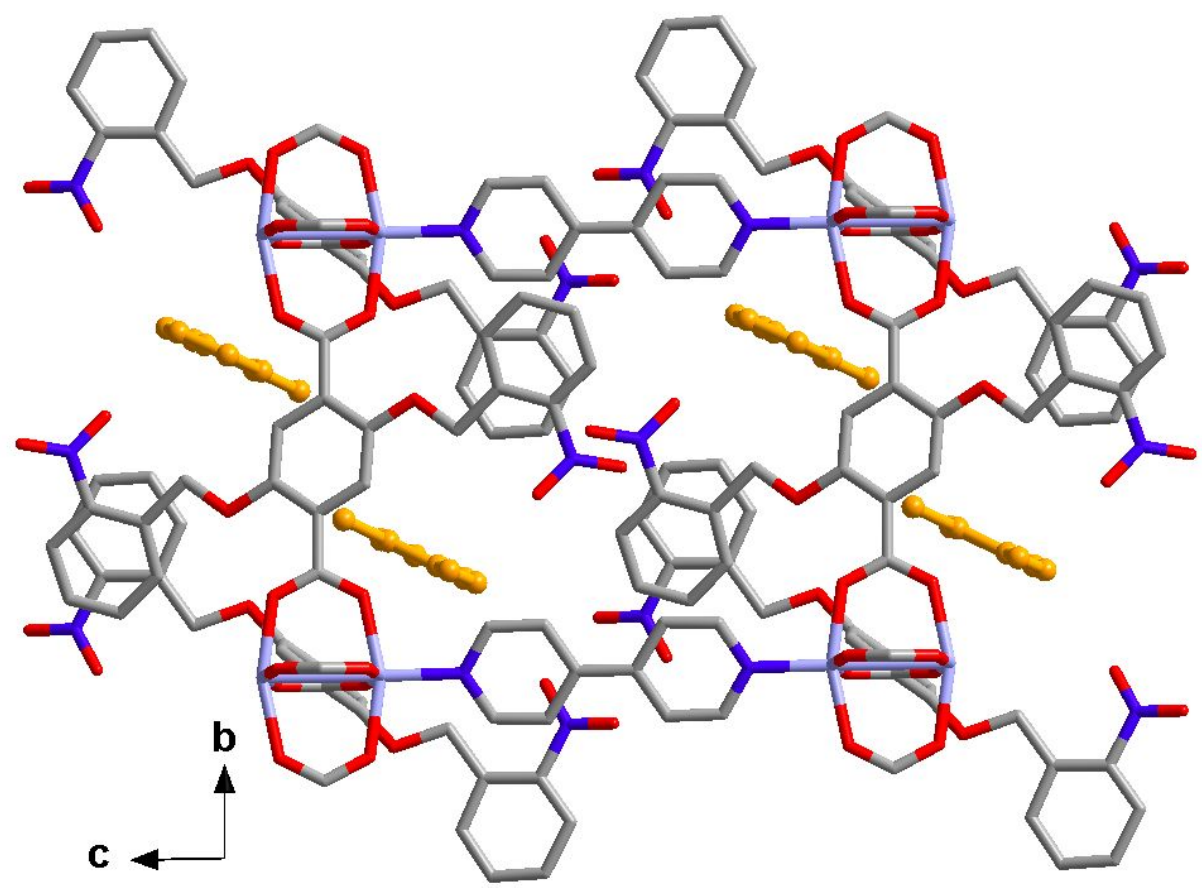

Figure S4. Side view of PCP-1-oX. 


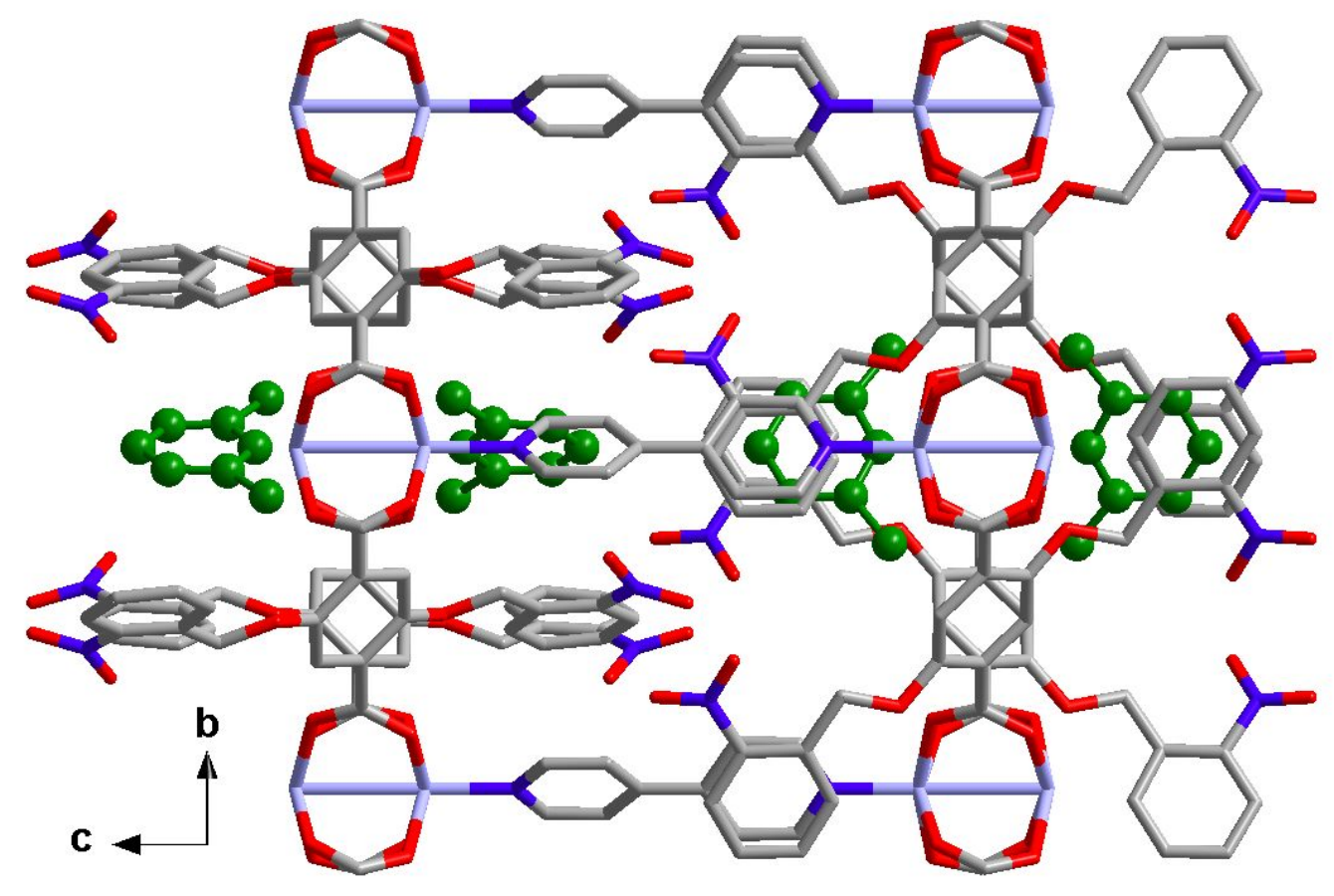

Figure S5. Side view of PCP-1-mX. 


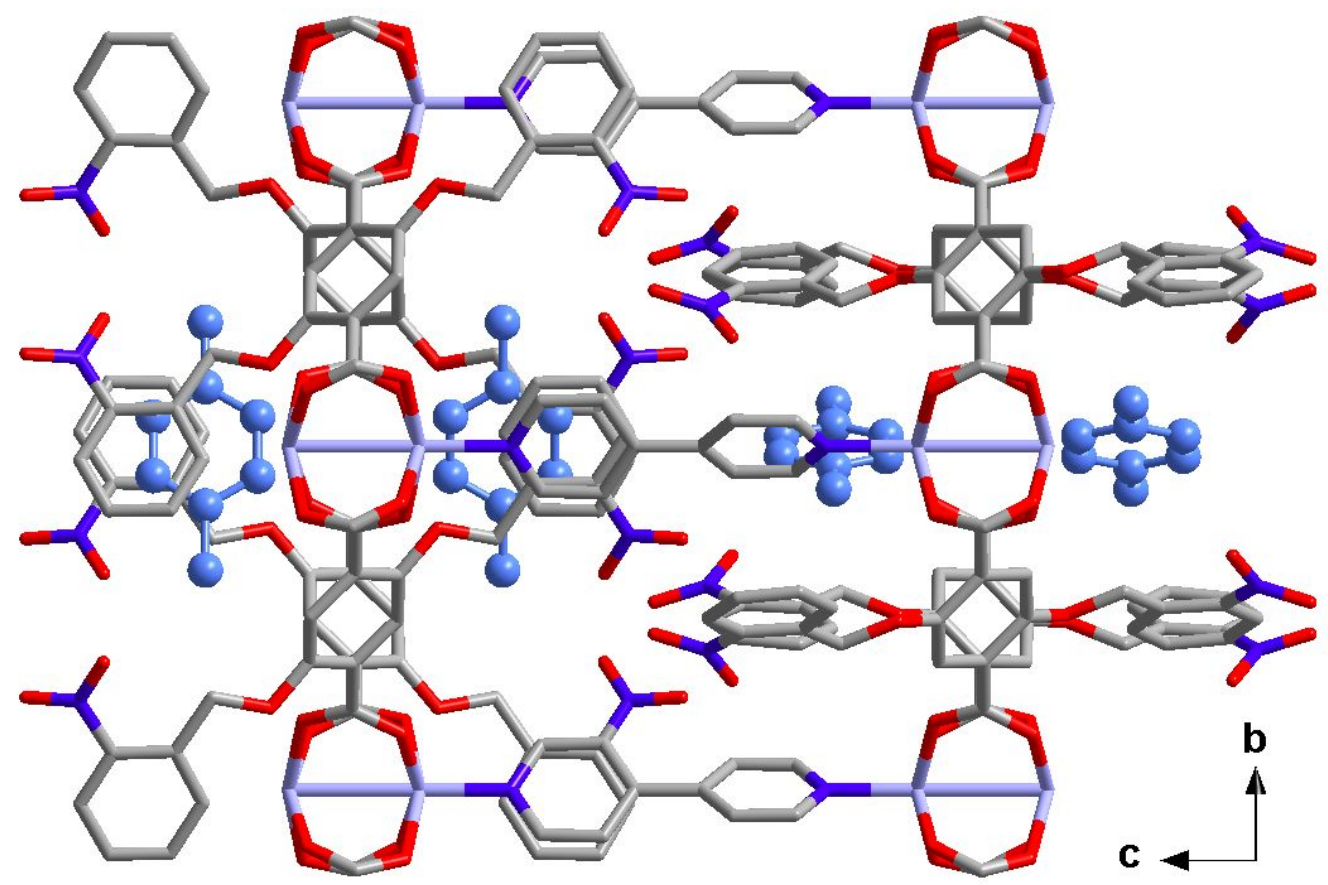

Figure S6. Side view of PCP-1-pX. 


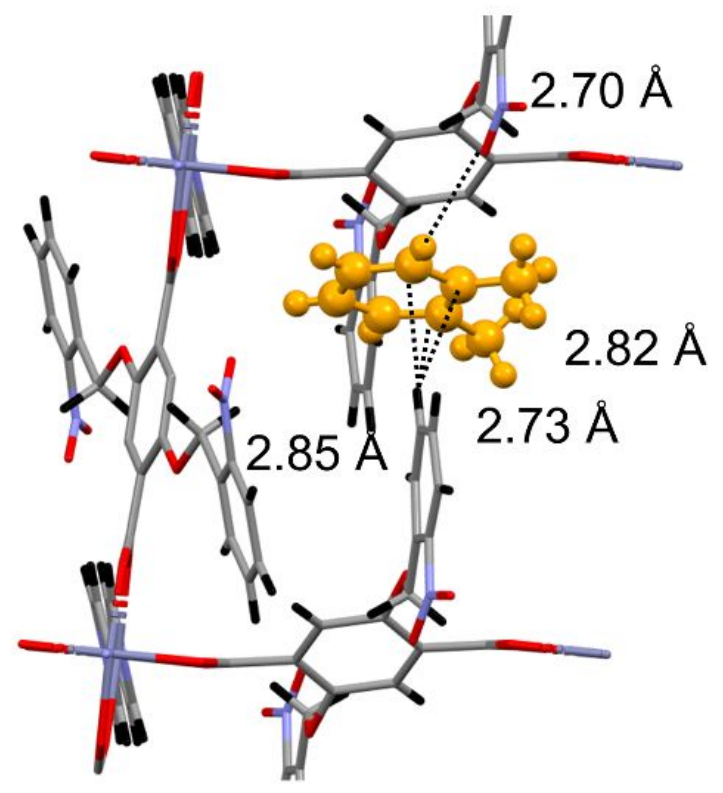

Figure S7. Host-guest interaction in PCP-1-oX. 


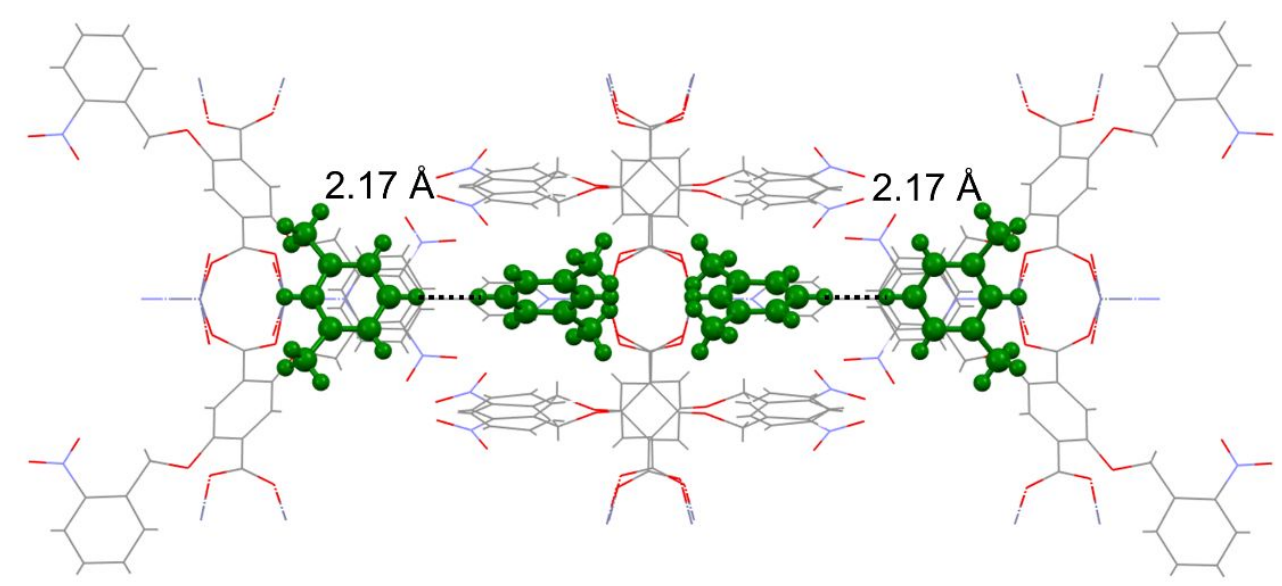

Figure S8. Guest-guest interaction in PCP-1-mX. 


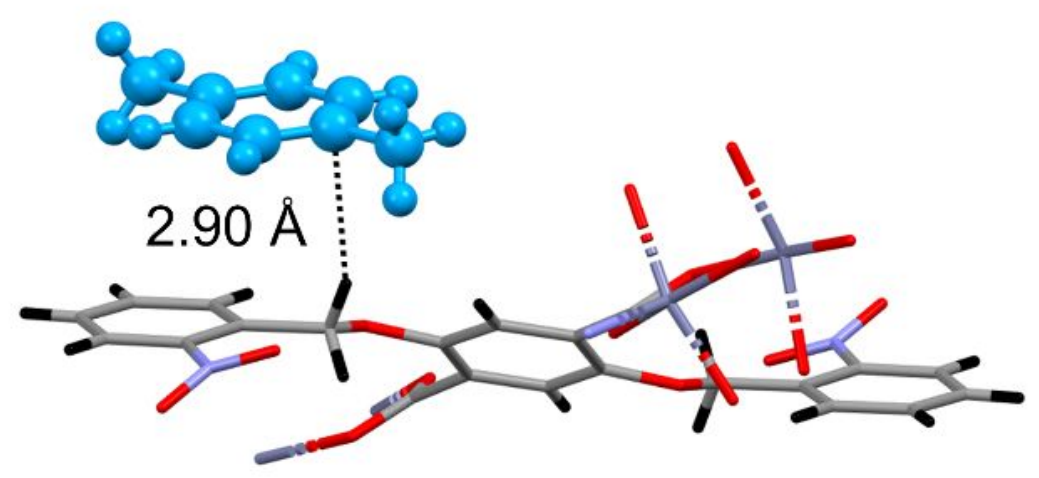

Figure S9. Host-guest interaction in PCP-1-pX. 


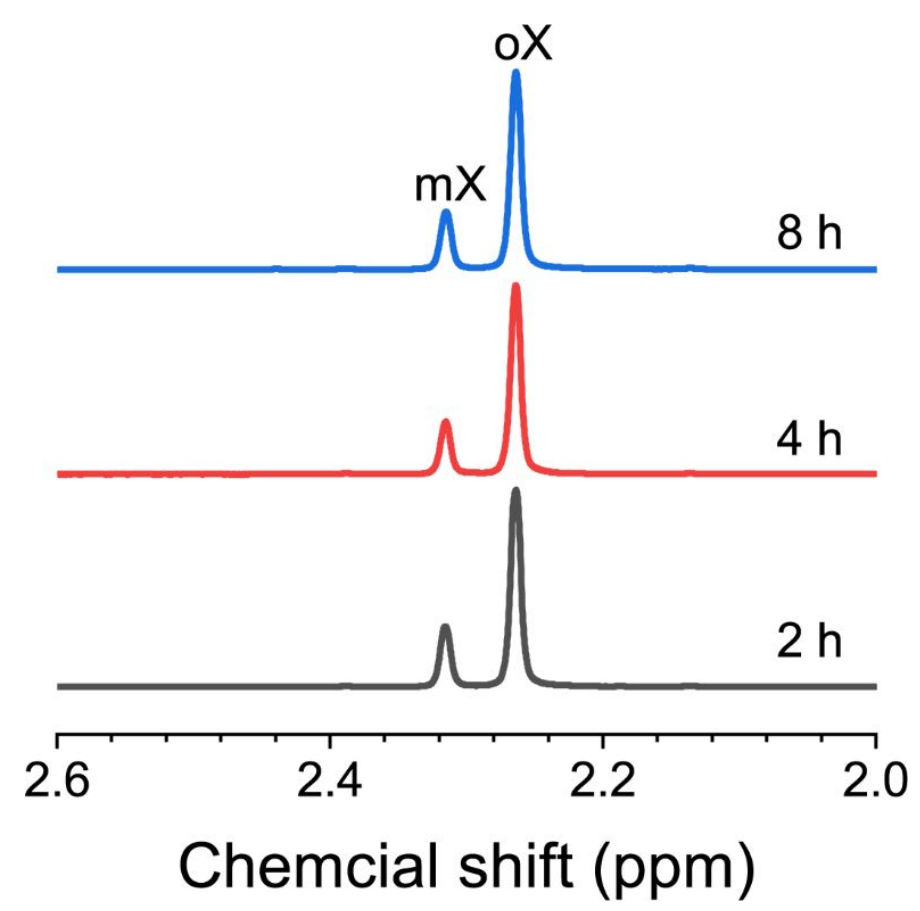

Figure S10. ${ }^{1} \mathrm{H}$ NMR spectra of PCP-1-act extracting $\mathrm{CDCl}_{3}$ after $\mathrm{OX}$ and $\mathrm{mX}$ binary mixture adsorption. 


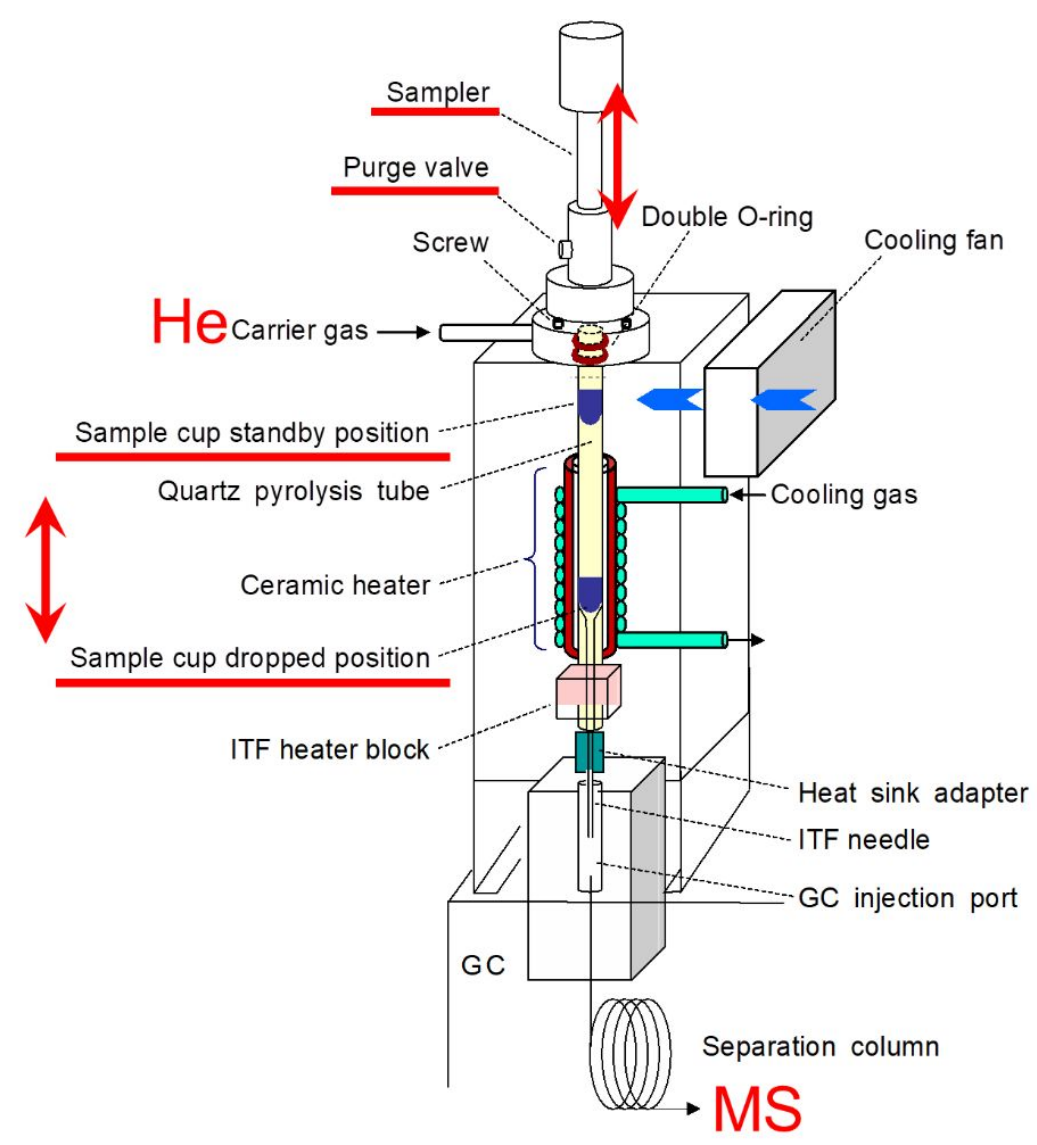

Figure S11. The Pyrolyzer of Py-GC-MS 


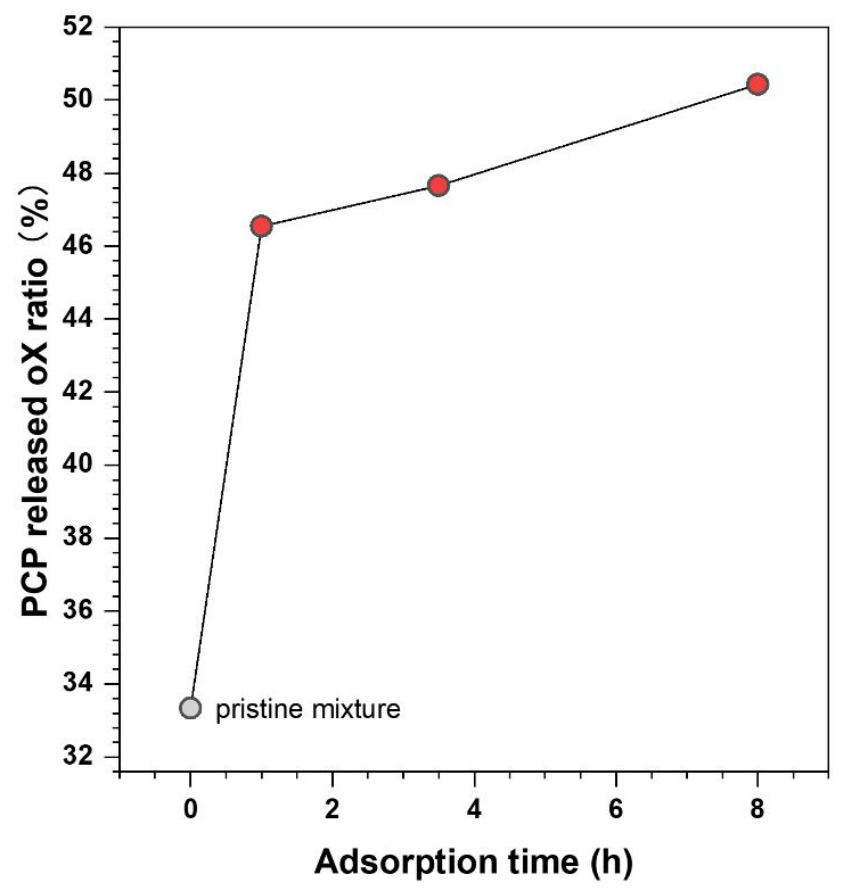

Figure S12. oX adsorption ratio from ternary isomer mixture along time monitored by Py-GC-MS. 


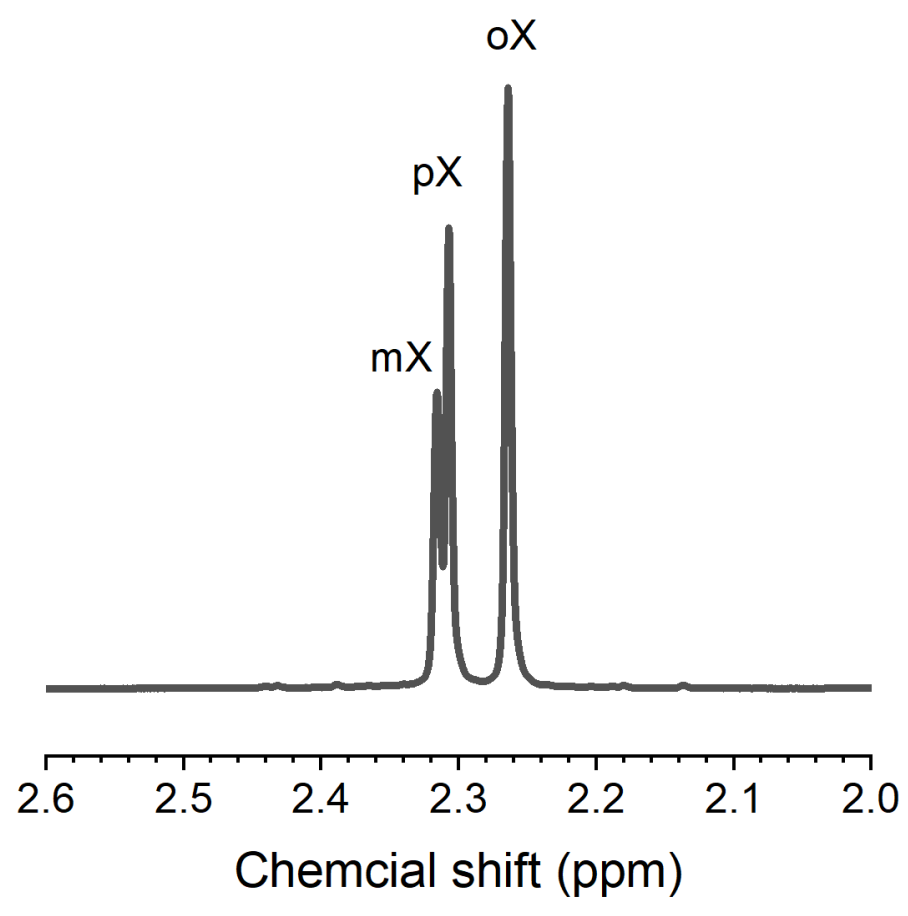

Figure S13. ${ }^{1} \mathrm{H}$ NMR spectrum of PCP-1-act extracting $\mathrm{CDCl}_{3}$ after $\mathrm{oX}, \mathrm{mX}$ and $\mathrm{pX}$ ternary mixture adsorption. 


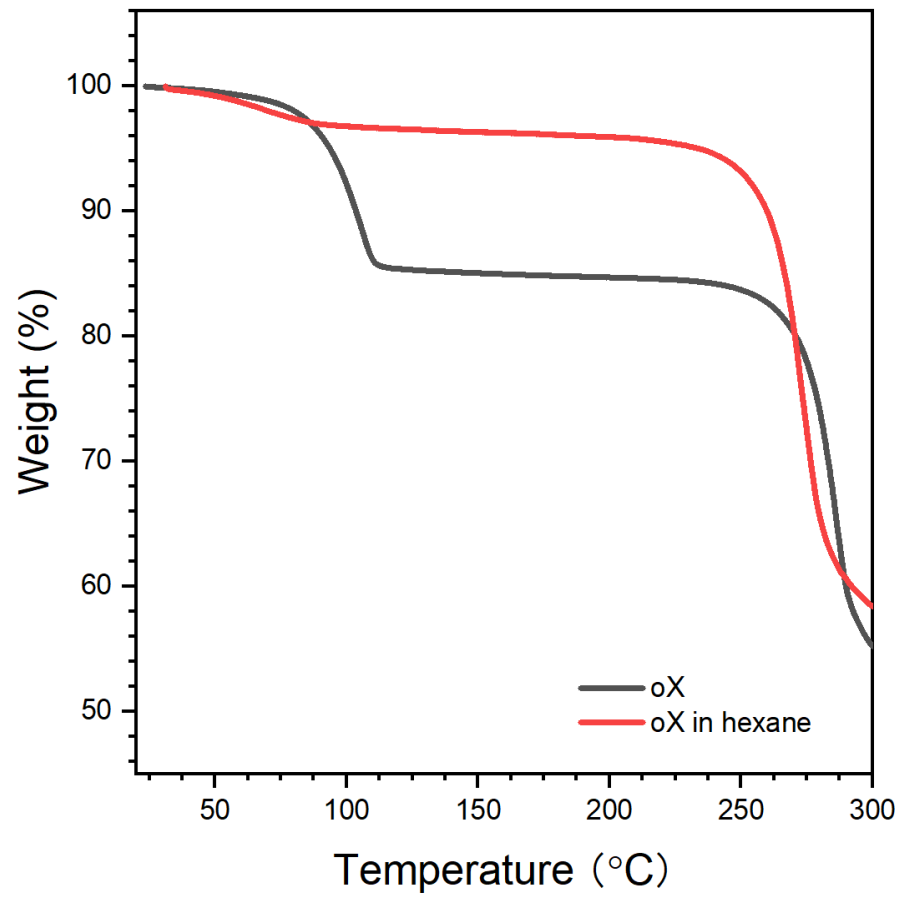

Figure S14. TGA analysis of PCP after adsorption in 1M oX-hexane solution. 


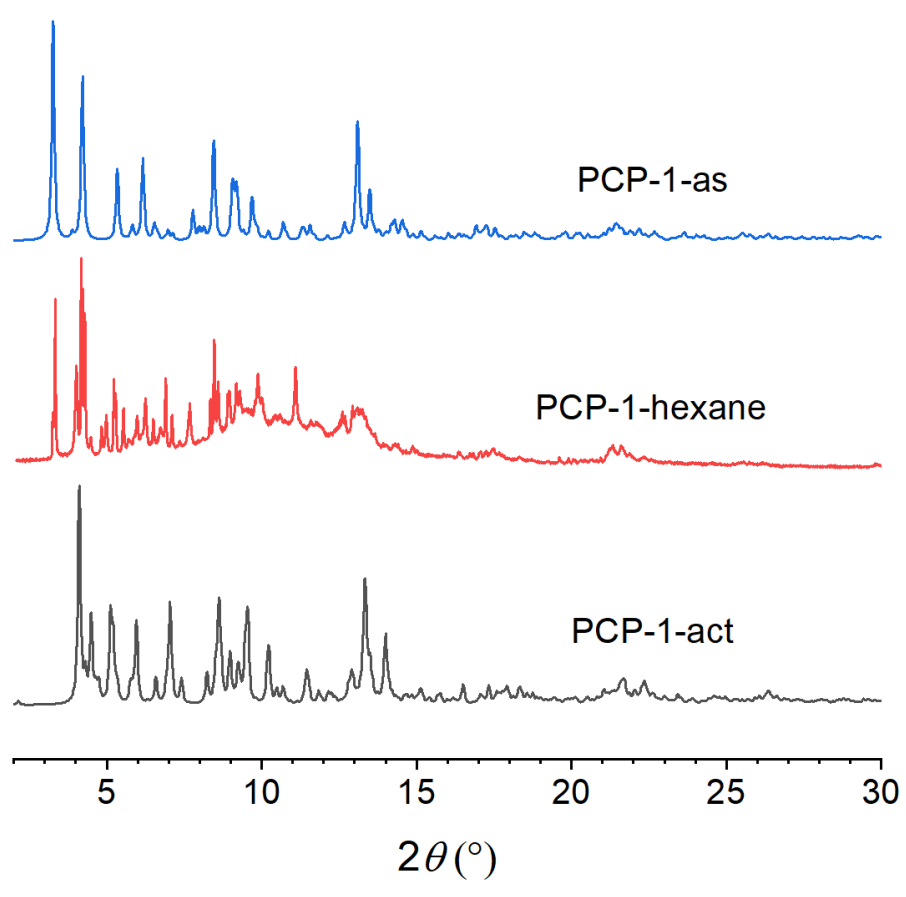

Figure 15 . Synchrotron PXRD patterns (0.79967 $\AA$ ) of PCP-1-act immersing in hexane (PCP-1-hexane). The PXRD pattern of PCP-1-hexane was measured by sealing activated PCP-1 (PCP-1-act) power and hexane together in a glass capillary. 


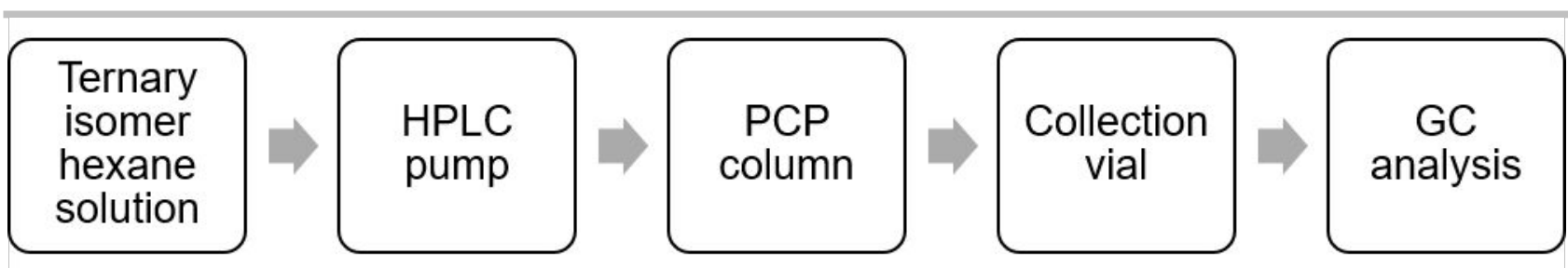

Figure S16. column-HPLC instrument. 


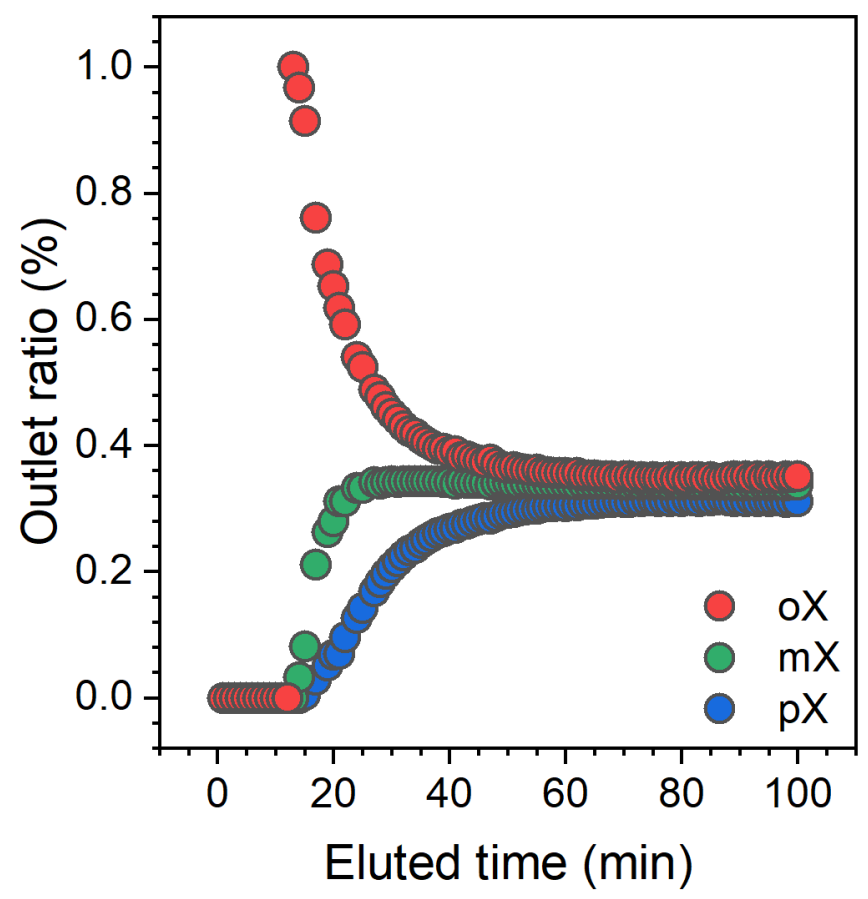

Figure S17. Ternary equimolar xylene isomer (100 mM) breakthrough experiment on PCP-1-act column using hexane as eluant. 


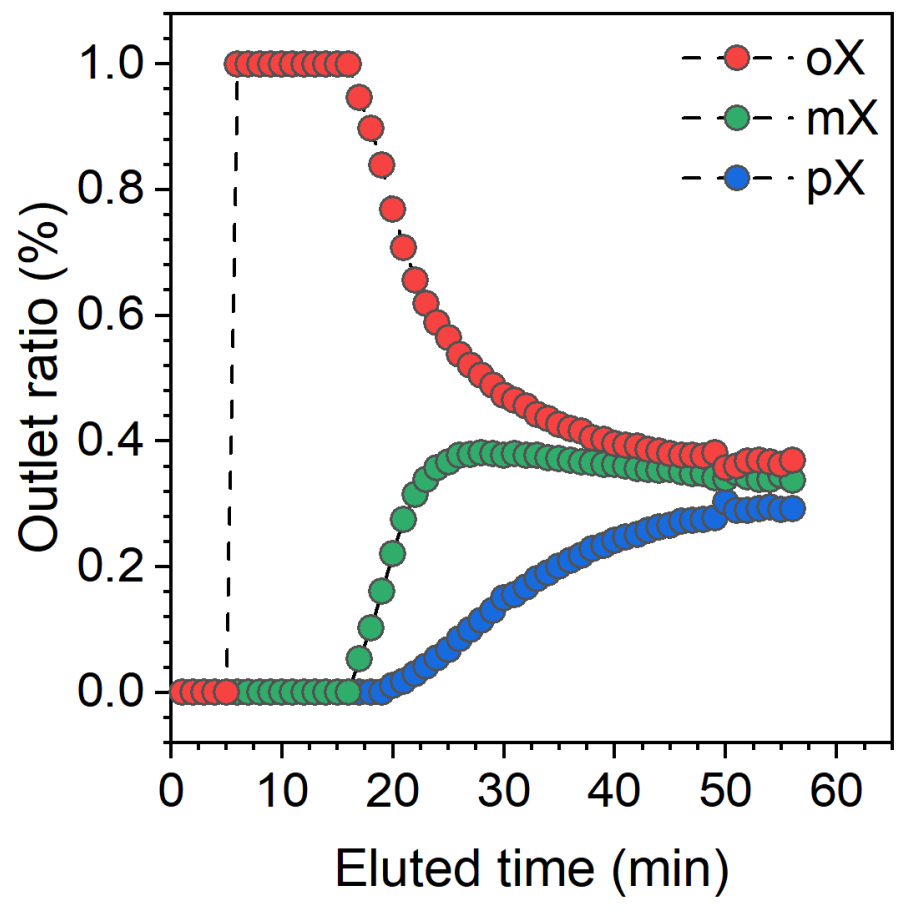

Figure S18. the $12^{\text {th }}$ cycle ternary equimolar xylene isomer $(15 \mathrm{mM})$ breakthrough experiment on PCP-1-act column using hexane as eluant 


\section{Supporting Table}

Table S1. Physical properties of xylene isomers ${ }^{3}$

\begin{tabular}{cccc}
\hline & oX & $\mathbf{m X}$ & $\mathbf{p X}$ \\
\hline P0 at 298 K $(\mathrm{kPa})$ & 1.16 & 1.46 & 1.54 \\
Boling point $\left({ }^{\circ} \mathrm{C}\right)$ & 144.4 & 139.1 & 138.4 \\
Molecular dimension $\left(\AA^{3}\right)$ & $7.267 \times 3.834 \times 7.826$ & $8.994 \times 3.949 \times 7.315$ & $6.618 \times 3.810 \times 9.146$ \\
MIN-1 $(\AA)$ & 3.834 & 3.949 & 3.810 \\
MIN-2 $(\AA)$ & 2.269 & 7.258 & 6.618 \\
\hline
\end{tabular}




\begin{tabular}{|c|c|c|c|c|}
\hline PCP & 1-act & $1-0 X$ & $1-m x$ & $1-p X$ \\
\hline Formula & $\mathrm{C} 54 \mathrm{H} 36 \mathrm{~N} 6 \mathrm{O} 20 \mathrm{Zn} 2$ & C35H28N3O10Zn1 & $\mathrm{C} 35 \mathrm{H} 28 \mathrm{~N} 3 \mathrm{O} 10 \mathrm{Zn} 1$ & $\mathrm{C} 35 \mathrm{H} 28 \mathrm{~N} 3010 \mathrm{Zn} 1$ \\
\hline$M_{t}$ & 1219.63 & 715.97 & 715.97 & 715.97 \\
\hline Crystal system & triclinic & triclinic & tetragonal & tetragonal \\
\hline Space group & $P \overline{1}$ (no. 2) & $P \overline{1}$ (no. 2) & $P 4_{2} / n b c($ no. 133$)$ & $P 4_{2} /$ nbc (no. 133) \\
\hline$a(\AA)$ & $10.764(2)$ & $10.8462(13)$ & $15.3684(3)$ & $15.3689(2)$ \\
\hline$b(\AA)$ & $11.671(2)$ & $10.9618(13)$ & $15.3684(3)$ & $15.3689(2)$ \\
\hline$c(\AA)$ & $27.444(3)$ & $13.9807(16)$ & $28.0526(7)$ & $28.1065(6)$ \\
\hline$\alpha\left({ }^{\circ}\right)$ & $87.73(1)$ & $90.699(9)$ & 90 & 90 \\
\hline$\beta\left({ }^{\circ}\right)$ & $82.74(1)$ & $93.916(9)$ & 90 & 90 \\
\hline$Y\left({ }^{\circ}\right)$ & $72.71(1)$ & $98.294(10)$ & 90 & 90 \\
\hline$V\left(\AA^{3}\right)$ & $2551.4(7)$ & $1640.6(3)$ & $6625.7(3)$ & 6638.84 \\
\hline$Z$ & 2 & 2 & 8 & 8 \\
\hline Temperature (K) & 100 & 100 & 98 & 123 \\
\hline Calcd Density $\left(\mathrm{g} / \mathrm{cm}^{3}\right)$ & 1.588 & 1.447 & 1.436 & 1.433 \\
\hline$\mu\left(\mathrm{mm}^{-1}\right)$ & 1.029 & 0.812 & 0.805 & 0.803 \\
\hline$F(000)$ & 1244 & 736 & 2952.0 & 2952.0 \\
\hline $\begin{array}{c}\text { Crystal } \\
\text { dimension }\left(\mathrm{mm}^{3}\right)\end{array}$ & $0.04 \times 0.05 \times 0.10$ & $0.04 \times 0.04 \times 0.10$ & $0.04 \times 0.05 \times 0.10$ & $0.02 \times 0.03 \times 0.05$ \\
\hline Goodness-of-Fit & 1.048 & 1.299 & 1.101 & 1.043 \\
\hline$R_{1}(I>2.0 \sigma(I))$ & 0.1206 & 0.1228 & 0.0674 & 0.0444 \\
\hline$w R_{2}$ (all data) & 0.2481 & 0.3405 & 0.1792 & 0.1080 \\
\hline $\begin{array}{l}\text { CCDC deposition } \\
\text { number }\end{array}$ & 2044101 & 2081400 & 2081398 & 2081399 \\
\hline
\end{tabular}


Table S3. The observed close contact distances in the crystal structures of xylene loading PCPs.

\begin{tabular}{|c|c|c|c|}
\hline PCP & $1-0 X$ & $1-m x$ & $1-p X$ \\
\hline Host-guest п-т stacking & $3.88 \AA$ & $4.22 \AA$ & $4.13 \AA$ \\
\hline Host-guest interaction & $\begin{array}{l}\mathrm{C} \ldots \mathrm{H}=2.73 \AA \\
\mathrm{C} \ldots \mathrm{H}=2.82 \AA \\
\mathrm{C} \ldots \mathrm{H}=2.85 \AA \\
\mathrm{O} \ldots \mathrm{H}=2.70 \AA\end{array}$ & - & $\mathrm{C} \ldots \mathrm{H}=2.90 \AA$ \\
\hline Guest-guestinteraction & - & $\mathrm{H} \ldots \mathrm{H}=2.17$ & - \\
\hline
\end{tabular}




\section{References}

[1] Sheldrick, G. M. Acta Cryst. 2008, A 64, 112-122.

[2] Wang P., Otake K.-i., Hosono N., Kitagawa S., Angew. Chem. Int. Ed. 2021, 60, 7030 -7035.

[3] Li X., Wang J., Bai N. et al. Nat. Commun. 2021, 11, 4280. 